\title{
An earlier and more confident diagnosis of idiopathic pulmonary fibrosis
}

\author{
Roland M. du Bois
}

ABSTRACT: A diagnosis of idiopathic pulmonary fibrosis (IPF) has serious implications for the affected individuals, who have a $50 \%$ likelihood of dying within 2-3 yrs, an outcome which is worse than many cancers. A swift and accurate diagnosis is imperative, especially as commencing treatment at a relatively early disease stage may have the greatest impact on reducing disease progression.

The 2011, IPF guidelines provide updated and simplified IPF diagnostic criteria that may facilitate making a more confident diagnosis. The key investigational tool is high-resolution computed tomography (HRCT). In the presence of the four classical features, that together accurately identify a usual interstitial pneumonia (UIP) pattern, a definitive diagnosis of IPF can be made. When HRCT honeycombing is absent, even in the presence of all other features including traction bronchiectasis, the guidelines provide no designation for this constellation of features that many clinicians and radiologists would regard as consistent with UIP.

The diagnostic algorithm suggested by the 2011 guidelines emphasises the importance of multidisciplinary discussion between clinicians, radiologists and pathologists to improve diagnostic confidence. The course of disease in IPF is unpredictable, but the importance of an early diagnosis is clear, as individuals with less severe lung function abnormalities have a better prognosis.

KEYWORDS: Diagnostic criteria, early diagnosis, high-resolution computed tomography, idiopathic pulmonary fibrosis, usual interstitial pneumonia

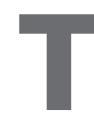
he most common and most lethal type of idiopathic interstitial pneumonia (IIP) is idiopathic pulmonary fibrosis (IPF), which accounts for $\sim 55 \%$ of lung diseases classified as IIPs [1-5]. The disease is uncommon in people aged $<50$ yrs [3]. Disease prevalence in the European Union is approximately $120,000[6,7]$, and it has been estimated that approximately 40,000 new cases will be diagnosed with IPF each year in Europe [6]. The prognosis for individuals with IPF is poor, far worse than nonspecific interstitial pneumonia and other IIPs (fig. 1) [8], with a 5-yr survival rate that is worse than several types of cancer (fig. 2) [8, 9]. Diagnosis of IPF requires precision and a multidisciplinary approach [2]. Indeed, an early and accurate diagnosis of IPF is critical for a better outcome, especially with the advent of new specific treatments for this disease.

\section{MAKING THE DIAGNOSIS}

Diagnostic criteria of $\mathbf{2 0 0 0}$

Previously, in the absence of surgical lung biopsy (SLB), the presence of all four major criteria and three out of four minor criteria were required in order to determine that the diagnosis was consistent with IPF $[1,10]$. The four major criteria were: 1) exclusion of other known causes of interstitial lung disease (ILD), such as certain drug toxicities, environmental exposures and connective tissue diseases; 2) abnormal lung function studies that include evidence of restriction (reduced vital capacity, often with an increased forced expiratory volume in $1 \mathrm{~s} /$ forced vital capacity ratio) and impaired gas exchange (increased alveolar-arterial oxygen tension difference with rest or exercise or decreased diffusing capacity of the lung for carbon monoxide); 3) high-resolution computed tomography (HRCT) scans showing bibasilar reticular abnormalities, with minimal ground-glass opacities; and 4) transbronchial lung biopsy (TBB) or bronchoalveolar lavage (BAL) showing no features to support an alternative diagnosis.

The four minor criteria were: 1) age >50 yrs; 2) insidious onset of otherwise unexplained dyspnoea on exertion; 3) duration of illness of
CORRESPONDENCE

R.M. du Bois

Imperial College London

London

SW7 2AZ

UK

E-mail: ron@du-bois.co.uk

Received:

Feb 032012

Accepted after revision:

Feb 202012

PROVENANCE

Publication of this peer-reviewed

article was sponsored by InterMune International AG, Muttenz,

Switzerland (article sponsor, European Respiratory Review issue 124). 


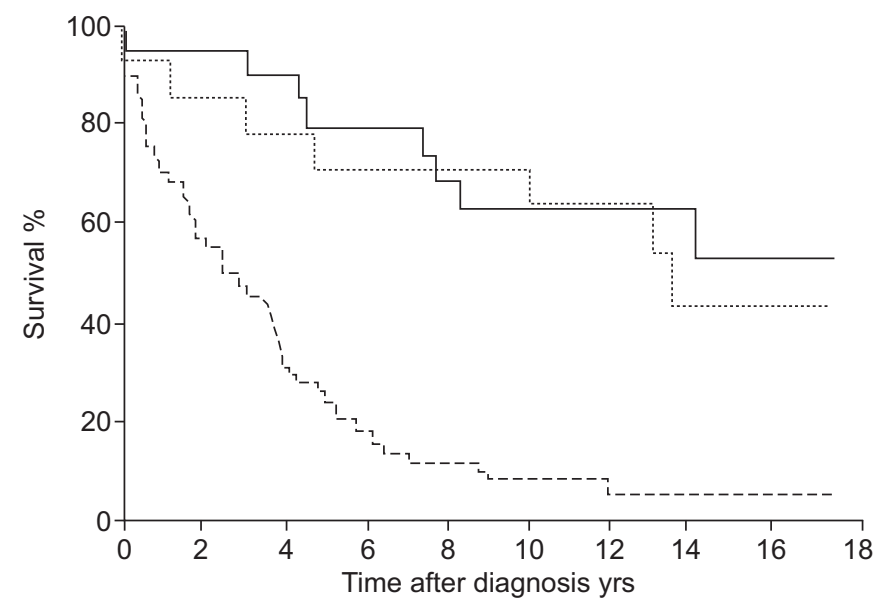

FIGURE 1. Prognosis of idiopathic pulmonary fibrosis is worse than nonspecific interstitial pneumonia and other idiopathic interstitial pneumonias - - other idiopathic interstitial pneumonias; $\cdots . . .$. . nonspecific interstitial pneumonias; - - - - -: idiopathic pulmonary fibrosis. Reproduced from [8] with permission from the publisher.

$\geqslant 3$ months; and 4) bibasilar, inspiratory crackles (dry or "velcro" type in quality).

\section{Diagnostic criteria of 2011}

In 2011, the American Thoracic Society (ATS), the European Respiratory Society (ERS), the Japanese Respiratory Society (JRS) and the Latin-American Thoracic Society (ALAT) issued an official joint statement providing guidance for the diagnosis and management of IPF [2]. These guidelines include a revision of the diagnostic criteria: 1) exclusion of other known causes of ILD (e.g. domestic and occupational environmental exposures, connective tissue disease and drug toxicity); 2) the presence of a usual interstitial pneumonia (UIP) pattern on

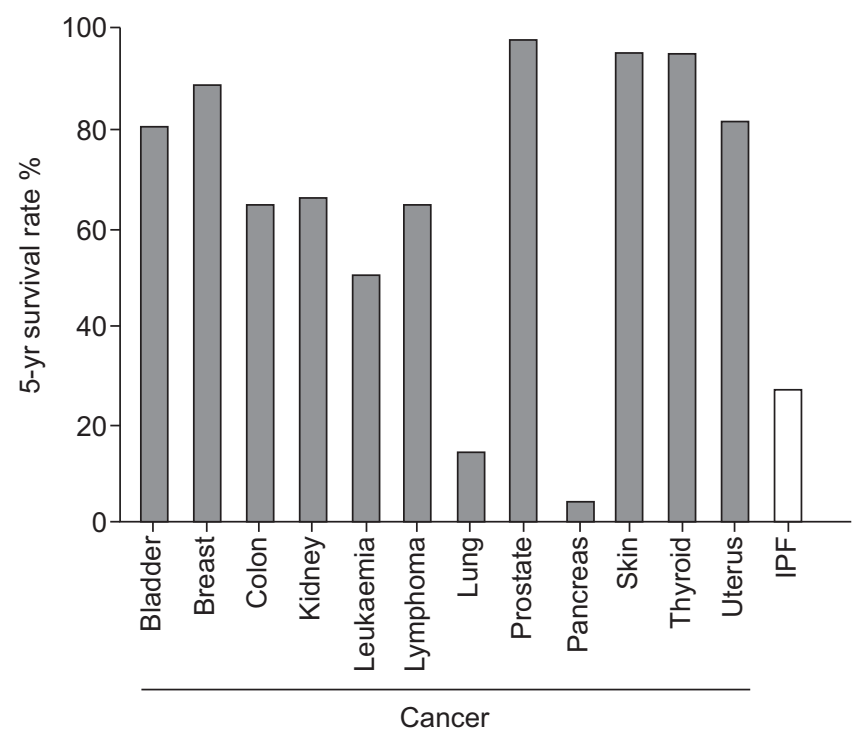

FIGURE 2. 5-yr survival rate of idiopathic pulmonary fibrosis (IPF) is worse than several types of cancer. Data taken from $[8,9]$.

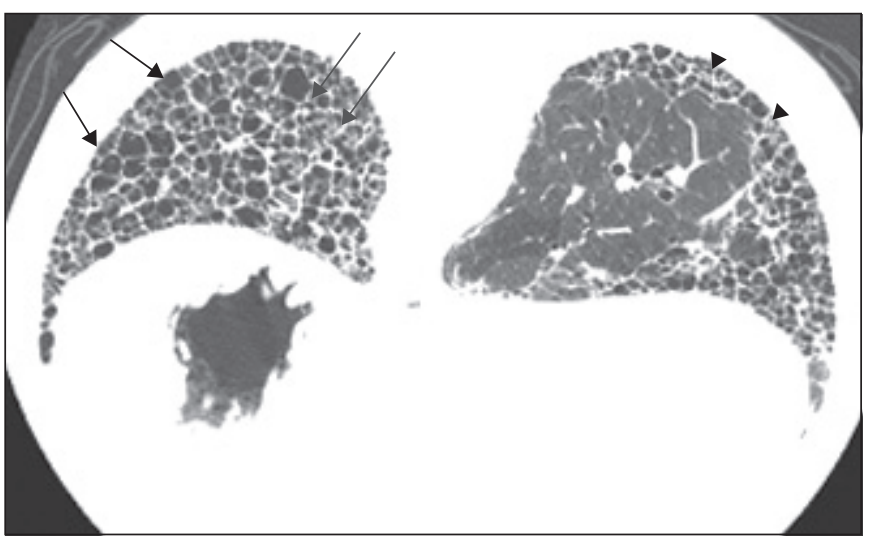

FIGURE 3. High-resolution computed tomography image of a typical usual interstitial pneumonia pattern. Black arrows: extensive honeycombing; grey arrows: traction bronchiectasis; arrowheads: reticular opacities.

HRCT scans in individuals not subjected to SLB; and 3) specific combinations of HRCT and SLB pattern in individuals subjected to SLB.

The previous guidelines using major and minor criteria for the clinical (i.e. non-pathological) diagnosis of IPF have been discarded, as it is now clear that, in an appropriate clinical setting, the presence of a classical UIP pattern on the HRCT scan is sufficient for a diagnosis of IPF to be made [2].

\section{Radiological features of IPF}

The features of a classical UIP pattern on HRCT require: 1) the presence of subpleural abnormalities, predominantly at the bases; 2) reticular abnormality; 3) honeycombing with or without traction bronchiectasis (fig. 3); and 4) the absence of features that are inconsistent with a UIP pattern (upper or middle lobe predominance, peribronchovascular predominance, extensive ground-glass abnormality (greater than reticular abnormality), profuse micronodules, discrete multiple cysts away from areas of honeycombing, diffuse mosaic attenuation/air trapping, or consolidation in bronchopulmonary segment(s)/lobe(s) [2, 11-13]). If honeycombing with or without traction bronchiectasis is absent then the diagnosis using HRCT can only be "possible". Notably, for those individuals with HRCT scan features falling into the category of "possible UIP pattern", or where there are atypical findings, further diagnostic evaluation is required (table 1) [2].

The radiological guidelines do, however, fall short in one circumstance. There is no category for individuals whose HRCT scan shows no honeycombing but do show all other UIP features, including traction bronchiectasis. Should this individual be classified as having only "possible disease" or characterised in some other way? At present, there is no term for this constellation of features that are not uncommonly seen in clinical practice, especially in those aged $>60 \mathrm{yrs}$, in patients with IPF. This deficiency needs to be remedied, perhaps with a "consistent with UIP pattern" category.

\section{Histopathological features of IPF}

The histopathological pattern of UIP is characteristically heterogeneous, predominantly subpleural, basal, bilateral and 
TABLE 1 High-resolution computed tomography criteria for usual interstitial pneumonia (UIP)

\begin{tabular}{|c|c|c|}
\hline UIP pattern ${ }^{\#}$ & Possible UIP pattern $\pi$ & Inconsistent with UIP pattern ${ }^{+}$ \\
\hline Subpleural, basal predominance & Subpleural, basal predominance & Upper or mid-lung predominance \\
\hline $\begin{array}{l}\text { Honeycombing with or without traction } \\
\text { bronchiectasis? }\end{array}$ & $\begin{array}{l}\text { Absence of features listed as inconsistent with } \\
\text { UIP pattern (see third column) }\end{array}$ & $\begin{array}{l}\text { Extensive ground-glass abnormality (extent more than } \\
\text { reticular abnormality)? }\end{array}$ \\
\hline
\end{tabular}

patchy in appearance [2]. For a definitive diagnosis of UIP, the following features are required to be present: 1) marked fibrosis/architectural distortion with or without honeycombing in a predominantly subpleural/paraseptal distribution; 2) patchy involvement of lung parenchyma by fibrosis; and 3) presence of fibroblast foci. In addition, the absence of any features considered to be inconsistent with a UIP pattern is also essential (table 2).

\section{CHALLENGES IN DIAGNOSING IPF IN CLINICAL PRACTICE}

The main challenge in achieving an accurate diagnosis of IPF is determining whether or not SLB is really needed, as referral for biopsy may delay the diagnosis being made. Furthermore, SLB is generally used in a minority of individuals with IPF worldwide, largely because the risks of SLB may outweigh the benefits of establishing a secure diagnosis of IPF in some

\section{TABLE 2 Histopathological criteria for usual interstitial pneumonia (UIP)}

\begin{tabular}{|c|c|c|c|}
\hline UIP pattern"\# & Probable UIP pattern & Possible UIP pattern" & Not UIP pattern ${ }^{+}$ \\
\hline $\begin{array}{l}\text { Evidence of marked fibrosis/architectural } \\
\text { distortion } \pm \text { honeycombing in a } \\
\text { predominantly subpleural/paraseptal } \\
\text { distribution }\end{array}$ & $\begin{array}{l}\text { Evidence of marked fibrosis/architectural } \\
\text { distortion } \pm \text { honeycombing }\end{array}$ & $\begin{array}{l}\text { Patchy or diffuse involvement } \\
\text { of lung parenchyma by fibrosis, } \\
\text { with or without interstitial } \\
\text { inflammation }\end{array}$ & Hyaline membranes ${ }^{\S}$ \\
\hline $\begin{array}{l}\text { Presence of patchy involvement } \\
\text { of lung parenchyma by fibrosis }\end{array}$ & $\begin{array}{l}\text { Absence of either patchy involvement or } \\
\text { fibroblastic foci, but not both }\end{array}$ & $\begin{array}{l}\text { Absence of other criteria for } \\
\text { UIP (see first column) }\end{array}$ & Organising pneumonia ${ }^{\S, f}$ \\
\hline Presence of fibroblast foci & $\begin{array}{l}\text { Absence of features against a diagnosis of } \\
\text { UIP suggesting an alternative diagnosis } \\
\text { (see fourth column) } \\
\text { OR } \\
\text { Honeycomb changes only \#\# }\end{array}$ & $\begin{array}{l}\text { Absence of features against a } \\
\text { diagnosis of UIP suggesting an } \\
\text { alternative diagnosis (see fourth } \\
\text { column) }\end{array}$ & Granulomas $^{f}$ \\
\hline $\begin{array}{l}\text { Absence of features against a diagnosis } \\
\text { of UIP suggesting an alternative } \\
\text { diagnosis (see fourth column) }\end{array}$ & & & $\begin{array}{l}\text { Marked interstitial inflammatory cell } \\
\text { infiltrate away from honeycombing } \\
\text { Predominant airway centred changes } \\
\text { Other features suggestive of an } \\
\text { alternative diagnosis }\end{array}$ \\
\hline
\end{tabular}

${ }^{\#}$ : all four features. ${ }^{\bullet}$ : all three features. ${ }^{+}$: any of the six features. ${ }^{\S}$ : can be associated with acute exacerbation of idiopathic pulmonary fibrosis. ${ }^{f}$ : an isolated or occasional granuloma and/or a mild component of organising pneumonia pattern may rarely be co-existing in lung biopsies with an otherwise UIP pattern. ${ }^{\# \#}$ : this scenario usually represents end-stage fibrotic lung disease where honeycombed segments have been sampled but where a UIP pattern might be present in other areas. Such areas are usually represented by overt honeycombing on high-resolution computed tomography and can be avoided by pre-operative targeting of biopsy sites away from these areas using high-resolution computed tomography. Reproduced from [2] with permission from the publisher. 


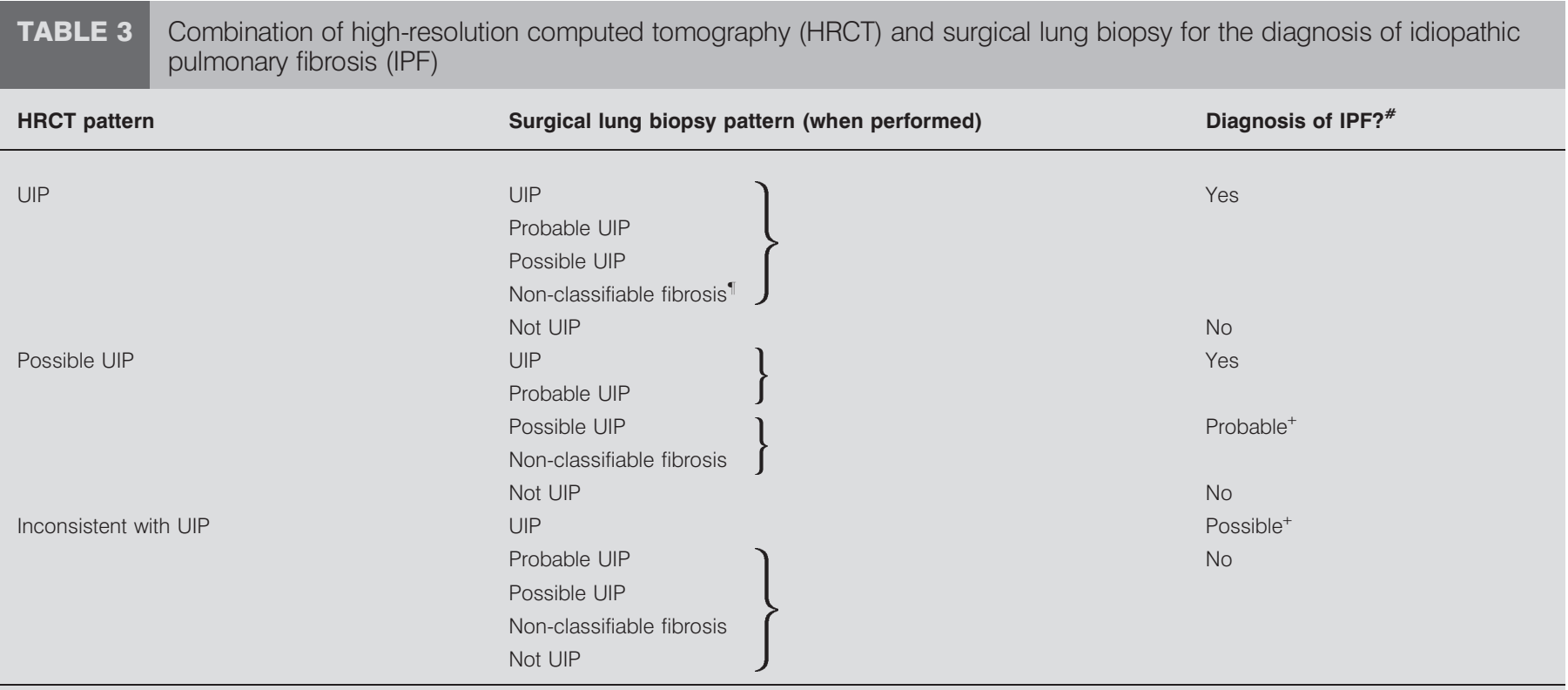

UIP: usual interstitial pneumonia. \#: the accuracy of diagnosis of IPF increases with multidisciplinary discussion (MDD). This is particularly relevant in cases in which the radiological and histopathological patterns are discordant, e.g. HRCT is inconsistent with UIP and histopathology is UIP. There are data to suggest that the accuracy of diagnosis is improved with MDD among interstitial lung disease experts compared with clinician specialists in the community setting; timely referral to interstitial lung disease experts is encouraged." : some biopsies may reveal a pattern of fibrosis that does not meet the criteria for UIP pattern and the other idiopathic interstitial pneumonias; these biopsies may be termed non-classifiable. ${ }^{+}$: MDD should include discussions of the potential for sampling error and a re-evaluation of adequacy of technique of HRCT. In cases with an "inconsistent with UIP" HRCT pattern and a "UIP" surgical lung biopsy pattern, the possibility of a diagnosis of IPF still exists and clarification by MDD among interstitial lung disease experts is indicated. Reproduced from [2] with permission from the publisher.

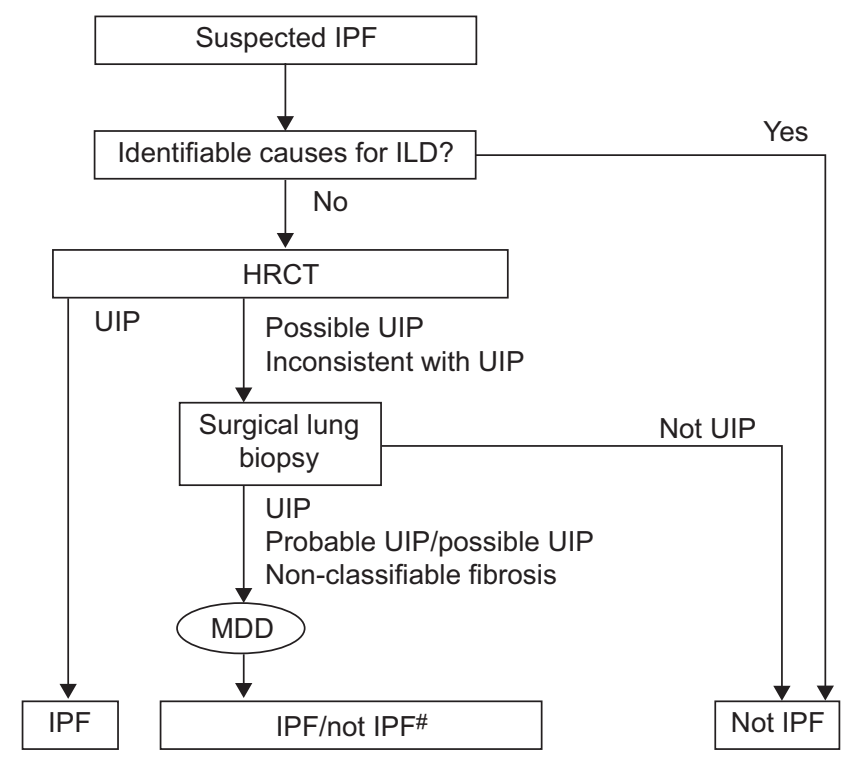

FIGURE 4. Diagnostic algorithm for idiopathic pulmonary fibrosis (IPF). Patients with suspected IPF (i.e. patients with unexplained dyspnoea on exertion and/or cough with evidence of interstitial lung disease (ILD)) should be carefully evaluated for identifiable causes of ILD. In the absence of an identifiable cause for ILD, a high-resolution computed tomography (HRCT) scan demonstrating usual interstitial pneumonia (UIP) pattern is diagnostic of IPF. In the absence of UIP pattern on HRCT, IPF can be diagnosed by the combination of specific HRCT and histopathological patterns. The accuracy of the diagnosis of IPF increases with multidisciplinary discussion (MDD) among ILD experts. \#: as per table 3. Reproduced from [2] with permission from the publisher. cases and the natural disinclination of most individuals to undergoing a surgical diagnostic procedure. In this context, individuals with HRCT scans that show reticular changes and traction bronchiectasis in the appropriate distribution can be diagnosed with almost $100 \%$ certainty as having IPF without SLB, especially if the individual is in the older age group [14], but the 2011 ATS/ERS/JRS/ALAT guidelines make no allowance for this category of "consistent with UIP" that would preclude those individuals needing biopsy. The current guidelines place these individuals in the same category as those who have neither honeycombing nor traction bronchiectasis, which is not logical.

It is a further failing of the 2011 guidelines that they do not provide sufficient guidance to address the question of when SLB should not be performed. The guidance provided states that the final decision regarding SLB should be tailored to the clinical situation of the individual and the surgical expertise available, but this is too vague.

The guidelines recommend that a multidisciplinary approach involving ILD specialists, radiologists and pathologists should be taken in the evaluation of suspected IPF. This approach has been shown to improve diagnostic accuracy and is now widely accepted to be the standard of care for the diagnosis of this and other ILDs. In this regard, the accuracy of IPF diagnosis increases with clinical, radiological and histopathological correlation and it is the combination of radiological and histopathological features taken in the clinical context that requires debate and informs decision making (table 3) [2]. There are often cases where individuals have discordant 
radiological and histopathological findings; for example, HRCT may be inconsistent with UIP, but the histopathology suggests UIP. It is in these cases particularly that the value of a multidisciplinary discussion becomes clear.

\section{DIAGNOSTIC ALGORITHM FOR IPF}

The 2011 guidelines provide a diagnostic algorithm for IPF (fig. 4) [2]. Individuals with suspected IPF (i.e. individuals with unexplained dyspnoea on exertion and/or cough with evidence of ILD) should be carefully evaluated for identifiable causes of ILD. In the absence of an identifiable cause for ILD, a HRCT demonstrating a UIP pattern is diagnostic of IPF. In the absence of a UIP pattern on HRCT, IPF can be diagnosed by the combination of specific HRCT and histopathological patterns. As noted above, the accuracy of the diagnosis increases with multidisciplinary discussion between pulmonologists, radiologists and pathologists experienced in the diagnosis of ILD [2].

\section{THE ROLE OF OTHER INVESTIGATIONS IN DIAGNOSIS AND PROGNOSIS}

Can methods less invasive than SLB, including BAL and TBB, be useful in the diagnosis of IPF? The short answer is no. BAL cellular analysis has been used for many years in the diagnosis of several ILDs. When evaluating individuals with suspected IPF, BAL is useful in excluding other conditions, especially chronic hypersensitivity pneumonitis, for which a diagnosis is suggested by a lymphocytosis $>40 \%$ [2]. A recent publication of retrospective data reported that $8 \%$ of individuals with a HRCT UIP pattern may have BAL features that would point to an alternative diagnosis to IPF [2, 15]. The 2011 guidelines recommend, however, that BAL cellular analysis should not be performed in the diagnostic evaluation of IPF in the majority of individuals, but may be appropriate for a minority. The evidence regarding whether or not BAL adds significantly improved diagnostic specificity is presently unclear [2].

TBB has been shown to be useful in the evaluation of conditions that are predominantly bronchocentric and that can be diagnosed from the relatively small samples that can be obtained by $\mathrm{TBB}$, for example sarcoidosis, especially in those relatively rare instances when the disease mimics IPF by having a UIP pattern on HRCT [16-18]. With regard to the use of TBB as a diagnostic procedure in IPF, its sensitivity and specificity are unknown, even when the biopsy material shows histological features of UIP $[2,19]$. Other problems include uncertainty about the best location to biopsy and the number of biopsies that should be taken. In view of these limitations, the 2011 guidelines do not recommend the use of TBB in the evaluation of IPF in the majority of individuals, but it may be helpful in a minority [2].

Serological testing for connective tissues disease is recommended for the diagnostic evaluation of IPF in the majority of individuals even in the absence of signs or symptoms of connective tissue disease, but may not be appropriate for all individuals. In addition, there is very low-quality evidence for the use of serological testing. However, it is valuable to exclude connective tissue disease, as this may present with a UIP pattern and it is recognised that this group of individuals generally have a better outcome than those who have idiopathic disease [20]. It is recommended that rheumatoid factor, anti-cyclic citrullinated peptide, and anti-nuclear antibody titre and pattern should form the screen and that other specific auto-antibodies should be sought only if any of the screening auto-antibodies are positive, or, obviously, if there were any subtle systemic features present that suggest early rheumatologic disease [2].

\section{CHALLENGES IN IPF CLINICAL MANAGEMENT}

IPF results in a progressive decline in lung function. The individual becomes incrementally housebound, oxygen dependent and, subsequently, wheelchair bound, resulting in death from respiratory failure or a complicating comorbidity. The clinical course of this disease is highly variable from individual to individual, as well as within the same individual. Many experience slow disease progression over numerous years, while some remain stable for long periods of time. Some individuals experience rapid progression and others experience episodes of acute respiratory worsening: acute exacerbation of IPF $[2,21]$. When IPF is first diagnosed, the individual has typically had asymptomatic disease progression for many years, which has resulted in incremental fibrosis. In advanced disease stages, the pathology of IPF features extensive, irreversible honeycombing and fixed fibrosis.

Early diagnosis of IPF is, therefore, a critical factor for improved prognosis, as individuals with early disease have less severe lung function abnormalities. The treatment of IPF is unlikely to improve lung function; indeed, the only approved treatment available for IPF, pirfenidone, can only reduce the decline in lung function [22], but may help to stabilise disease. No treatment is curative.

\section{CONCLUSIONS}

The 5-yr survival rates of individuals with IPF are generally poorer than those for many types of cancer. This accentuates the urgent need for improvements in diagnosing IPF swiftly and confidently. The earlier the diagnosis is made, the sooner therapy can be initiated, which, in turn, may reduce the decline in lung function and improve the overall prognosis. The recently published ATS/ ERS/JRS/ALAT guidelines are of some help in simplifying the diagnostic process, but are incomplete in terms of the role of HRCT-only diagnoses, and are unhelpful and inconsistent in regard to treatment recommendations. Despite these deficiencies, the guidelines provide an improvement on the version published in 2000 and should be a good platform for a more definitive document to be produced in the near future that will further aid the diagnostic process in this crippling disease. Multidisciplinary discussions between experts in pulmonology, radiology and pathology will remain the cornerstone to improving the confidence in diagnosing IPF early and accurately, and facilitating the early use of effective therapies as they emerge from the pipeline.

\section{STATEMENT OF INTEREST}

R.M. du Bois is, or has recently been, a paid consultant, steering committee member, and/or co-chair of a steering committee for Actelion, Bayer, Boehringer Ingelheim, GlaxoSmithKline, InterMune, Novartis and Merck.

\section{REFERENCES}

1 American Thoracic Society, European Respiratory Society. American Thoracic Society/European Respiratory Society International Multidisciplinary Consensus Classification of the Idiopathic Interstitial Pneumonias. Am J Respir Crit Care Med 2002; 165: 277-304.

2 Raghu G, Collard HR, Egan JJ, et al. An official ATS/ERS/JRS/ALAT statement: idiopathic pulmonary fibrosis: evidence-based guidelines 
for diagnosis and management. Am J Respir Crit Care Med 2011; 183: 788-824.

3 Meltzer EB, Noble PW. Idiopathic pulmonary fibrosis. Orphanet J Rare Dis 2008; 3: 8-22.

4 Collard HR, King TE Jr, Bartelson BB, et al. Changes in clinical and physiologic variables predict survival in idiopathic pulmonary fibrosis. Am J Respir Crit Care Med 2003; 168: 538-542.

5 Kim DS, Collard HR, King TE Jr. Classification and natural history of the idiopathic interstitial pneumonias. Proc Am Thorac Soc 2006; 3: 285-292.

6 Navaratnam V, Fleming KM, West J, et al. The rising incidence of idiopathic pulmonary fibrosis in the UK. Thorax 2011; 66: 462-467.

7 Orphanet Report Series. Prevalence of rare disease: bibliographic data. Number 1. 2011. www.orpha.net/orphacom/cahiers/docs/ GB/Prevalence_of_rare_diseases_by_alphabetical_list.pdf

8 Bjoraker JA, Ryu JH, Edwin MK, et al. Prognostic significance of histopathologic subsets in idiopathic pulmonary fibrosis. Am J Respir Crit Care Med 1998; 157: 199-203.

9 American Cancer Society. Cancer Facts and Figures 2009. Atlanta, American Cancer Society, 2010.

10 American Thoracic Society. Idiopathic pulmonary fibrosis: diagnosis and treatment. International consensus statement. American Thoracic Society (ATS), and the European Respiratory Society (ERS). Am J Respir Crit Care Med 2000; 161: 646-664.

11 Nishimura $K$, Kitaichi $M$, Izumi $T$, et al. Usual interstitial pneumonia: histologic correlation with high-resolution CT. Radiology 1992; 182: 337-342.

12 Johkoh T, Muller NL, Cartier Y, et al. Idiopathic interstitial pneumonias: diagnostic accuracy of thin-section CT in 129 patients. Radiology 1999; 211: 555-560.
13 Hansell DM, Bankier AA, Macmahon $\mathrm{H}$, et al. Fleischner Society: glossary of terms for thoracic imaging. Radiology 2008; 246: 697-722.

14 Fell CD, Martinez FJ, Liu LX, et al. Clinical predictors of a diagnosis of idiopathic pulmonary fibrosis. Am J Respir Crit Care Med 2010; 181: 832-837.

15 Ohshimo S, Bonella F, Cui A, et al. Significance of bronchoalveolar lavage for the diagnosis of idiopathic pulmonary fibrosis. Am J Respir Crit Care Med 2009; 179: 1043-1047.

16 Hunninghake GW, Zimmerman MB, Schwartz DA, et al. Utility of a lung biopsy for the diagnosis of idiopathic pulmonary fibrosis. Am J Respir Crit Care Med 2001; 164: 193-196.

17 Raghu G, Mageto YN, Lockhart D, et al. The accuracy of the clinical diagnosis of new-onset idiopathic pulmonary fibrosis and other interstitial lung disease: a prospective study. Chest 1999; 116: $1168-1174$.

18 Flaherty KR, Thwaite EL, Kazerooni EA, et al. Radiological versus histological diagnosis in UIP and NSIP: survival implications. Thorax 2003; 58: 143-148.

19 Berbescu EA, Katzenstein AA, Snow JL, et al. Transbronchial biopsy in usual interstitial pneumonia. Chest 2006; 129: 1126-1131.

20 Park JH, Kim DS, Park IN, et al. Prognosis of fibrotic interstitial pneumonia: idiopathic versus collagen vascular disease-related subtypes. Am J Respir Crit Care Med 2007; 175: 705-711.

21 Valeyre D. Towards a better diagnosis of idiopathic pulmonary fibrosis. Eur Respir Rev 2011; 20: 108-113.

22 Noble PW, Albera C, Bradford WZ, et al. Pirfenidone in patients with idiopathic pulmonary fibrosis (CAPACITY): two randomised trials. Lancet 2011; 377: 1760-1769. 